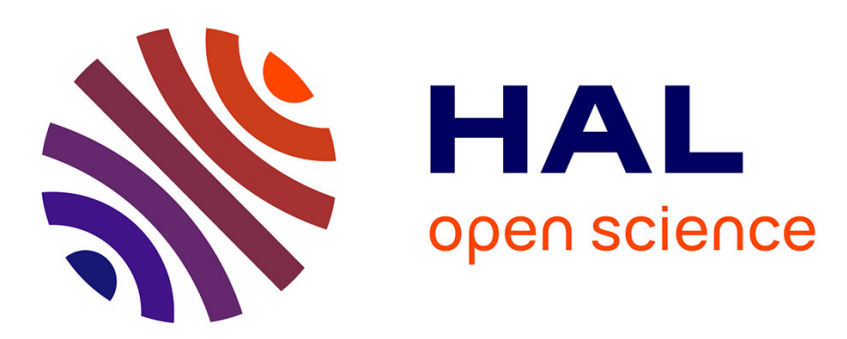

\title{
An indirect closed loop subspace identification method
}

Mathieu Pouliquen, Olivier Gehan, Eric Pigeon

\section{To cite this version:}

Mathieu Pouliquen, Olivier Gehan, Eric Pigeon. An indirect closed loop subspace identification method. IEEE Conference on Decision and Control, CDC'10, Dec 2010, Atlanta, United States. pp.4417 - 4422, 10.1109/CDC.2010.5717786 . hal-01059161

\section{HAL Id: hal-01059161 https://hal.science/hal-01059161}

Submitted on 29 Aug 2014

HAL is a multi-disciplinary open access archive for the deposit and dissemination of scientific research documents, whether they are published or not. The documents may come from teaching and research institutions in France or abroad, or from public or private research centers.
L'archive ouverte pluridisciplinaire HAL, est destinée au dépôt et à la diffusion de documents scientifiques de niveau recherche, publiés ou non, émanant des établissements d'enseignement et de recherche français ou étrangers, des laboratoires publics ou privés. 


\title{
An indirect closed loop subspace identification method
}

\author{
Mathieu Pouliquen, Olivier Gehan, Eric Pigeon \\ Control Group, GREYC CNRS UMR 6072 \\ ENSICAEN, 06 Bd du Marechal Juin \\ 14050 Caen Cedex, France \\ mathieu.pouliquen@greyc.ensicaen.fr
}

\begin{abstract}
In this paper, we consider a closed-loop subspace identification problem. An indirect method is developed using exogenous input and knowledge of a part of the controller impulse response. The idea is to extract dynamic of the plant from dynamic of the closed loop system. Two main result allows this double estimation. Only the deterministic behavior of the plant is considered in this paper. A simulation example is given to illustrate the performances of the present method.
\end{abstract}

\section{INTRODUCTION}

The identification of closed loop systems has received much interest for the last decades ([33], [34] [26], [10], [8], [11]) because for many industrial processes open loop experiments are prohibited (safety, stability, efficiency of operation, etc.). Due to the feedback control, a main difficulty in the identification of system operating in closed loop is the existence of a correlation between plant inputs and disturbances, thus different specific methods have been developed.

Recently there has been also an increasing interest in subspace identification algorithm as pointed out by the available contributions ([35], [9], [18], [31], [32], [6]). These methods have been mainly motivated by a set of interesting properties: the simplicity, the intrinsic numerical robustness and their straightforward application to multivariable systems. However, it is a fact that classical subspace methods fail when data are collected in closed loop experiments: these methods require the noise sequence to be orthogonal to the input sequence which is not the case with a feedback.

To overcome this difficulty, some particular subspace methods devoted to closed loop identification have been proposed. These methods are divided into three groups namely the direct approaches, the indirect approaches and the joint input-output approaches:

- In the direct approaches the identification is performed as in an usual open loop context up to a suitable data processing ([25], [7], [30], [15], [23], [24], [16], [2], [3], [13], [14], [4], [39], [12]);

- The indirect approaches are mainly based on an open loop identification of the control system sensitivity function using the system output and an external excitation input ([36]-[37], [27], [29], [28]);

- The joint input-ouput approaches use the system inputoutput behavior together with an external excitation input ([38], [17], [19], [21], [22]).
Some very attractive direct approaches have been analyzed in [2], [3], [4], [5] and [6]. These methods "may be regarded (...) as a significant step towards a satisfactory subspace identification algorithm working with closed-loop data" ([4]). Analyzing the behavior of the closed loop system our indirect approach is very different: a characteristic of the closed loop system is first obtained using projections of subspace, as is the case in lot of subspace identification algorithms. Then model of the plant is extracted making use the knowledge of the controller dynamic. Notice that the deterministic part of the plant only is considered here.

The paper is organized as follows. In section II, the closed loop identification problem considered in the paper is formulated. Several notations and assumptions used throughout the paper are also given. Section III is the highlight of the paper: subsection III-A is devoted to two main results and the proposed indirect subspace identification method is explained in Subsection III-B. In Section IV, a numerical simulation study is reported and finally, some concluding remarks are presented in Section V.

\section{PROBLEM FORMULATION AND NOTATION}

\section{A. Identification context}

Consider the identification of the closed loop system shown in Fig. 1 where $u(t) \in \mathbb{R}^{n_{u}}$ and $y(t) \in \mathbb{R}^{n_{y}}$ are respectively the input and the output of the plant which is expressed as:

$$
y(t)=G(q) u(t)+H(q) e(t)
$$

$G(q)$ is a proper transfer matrix of the deterministic part of the plant, $H(q)$ is an inversely stable square transfer matrix and $e(t) \in \mathbb{R}^{n_{y}}$ a white noise representing the innovation. A minimal state space model for (1) can be written in an innovation form as

$$
\left\{\begin{array}{cl}
x(t+1) & =A x(t)+B u(t)+K e(t) \\
y(t) & =C x(t)+D u(t)+e(t)
\end{array}\right.
$$

with $A \in \mathbb{R}^{n \times n}, B \in \mathbb{R}^{n \times n_{u}}, C \in \mathbb{R}^{n_{y} \times n}, D \in \mathbb{R}^{n_{y} \times n_{u}}$ and $K \in \mathbb{R}^{n \times n_{y}}$. $(A, C)$ is assumed to be observable and $(A, B)$ is assumed to be controllable. Note that $A$ should not be stable, which it indeed often is not in a closed loop context.

The control signal $u(t)$ is generated by $u(t)=r(t)-$ $C(q) y(t)$. The controller state space equations are defined as

$$
\left\{\begin{array}{cl}
x_{c}(t+1) & =A_{c} x_{c}(t)-B_{c} y(t) \\
u(t) & =C_{c} x_{c}(t)-D_{c} y(t)+r(t)
\end{array}\right.
$$




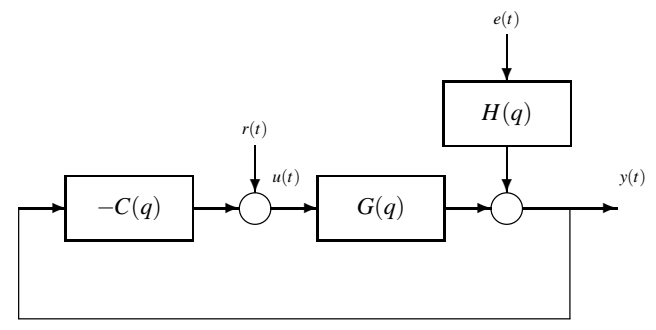

Fig. 1. Closed loop system

where $A_{c} \in \mathbb{R}^{n_{c} \times n_{c}}, B_{c} \in \mathbb{R}^{n_{c} \times n_{y}}, C_{c} \in \mathbb{R}^{n_{u} \times n_{c}}$ and $D_{c} \in$ $\mathbb{R}^{n_{u} \times n_{y}}$. The controller should not be stable. Here $r(t) \in \mathbb{R}^{n_{u}}$ is an exogenous input for identification.

The identification problem treated in this paper is stated as: find the order $n$ of the system and a realization of state space matrices $(A, B, C, D)$ given input, output and external excitation measurements. The knowledge of the first Markov parameters of the controller is also required but they can easily be estimated (see end of subsection III-A).

We make some usual assumptions throughout the paper:

- The closed loop system is internally stable.

- $r(t)$ and $e(t)$ are wide sense stationary zero mean processes and are second order ergodic.

- $r(t)$ satisfies persistent excitation conditions.

- $r(t)$ and $e(t)$ are mutually uncorrelated.

- The control system of Fig. 1 is assumed to be well posed. The well posed assumption of the control system ensures that $\left(I_{n_{u}}+D_{c} D\right)$ and $\left(I_{n_{y}}+D D_{c}\right)$ are non singular.

\section{B. Notations}

Standard subspace algorithms split the available data into block matrices: the input Hankel matrices $U_{p}$ and $U_{f}$ are defined as

$$
\left(\frac{U_{p}}{U_{f}}\right)=\left(\begin{array}{cccc}
u(t-p) & u(t-p+1) & \ldots & u(t-p+j-1) \\
u(t-p+1) & u(t-p+2) & \ldots & u(t-p+j) \\
\ldots & \ldots & \ldots & \ldots \\
u(t-1) & u(t) & \ldots & u(t+j-2) \\
\hline u(t) & u(t+1) & \ldots & u(t+j-1) \\
u(t+1) & u(t+2) & \ldots & u(t+j) \\
\ldots & \ldots & \ldots & \ldots \\
u(t+f-1) & u(t+f) & \ldots & u(t+f+j-2)
\end{array}\right)
$$

Similar definitions hold for $R_{f}, R_{p}, Y_{f}, Y_{p}, E_{f}$ and $E_{p}$. Subscripts $f$ and $p$ respectively stand for "future" and "past" and are user-defined indexes such that $f, p>n+n_{c} . j$ is the number of columns in the Hankel matrices. In subspace identification algorithm, it is assumed that there are long time series available i.e. $j \rightarrow \infty$. Due to the assumed ergodicity, the expected operator $\mathscr{E}\{$.$\} will be replaced by the operator$ $\mathscr{E}_{j}\{\cdot\}$ :

$$
\mathscr{E}_{j}\{.\}=\lim _{j \rightarrow \infty} \frac{1}{j}\{.\}
$$

(2) and (3) can be represented by the following basic subspace equations:

$$
\begin{aligned}
& \left\{\begin{array}{l}
X_{t}=A^{p} X_{t-p}+\Delta_{p}^{d} U_{p}+\Delta_{p}^{s} E_{p} \\
Y_{f}=\Gamma_{f} X_{t}+H_{f}^{d} U_{f}+H_{f}^{s} E_{f}
\end{array}\right. \\
& \left\{\begin{array}{l}
X_{t}^{c}=A_{c}^{p} X_{t-p}^{c}-\Delta_{p}^{c} Y_{p} \\
U_{f}=\Gamma_{f}^{c} X_{t}^{c}-H_{f}^{c} Y_{f}+R_{f}
\end{array}\right.
\end{aligned}
$$

where $X_{t-p}$ and $X_{t}$ are respectively the past and future state matrices of the plant:

$$
\begin{gathered}
X_{t-p}=\left(\begin{array}{llll}
x(t-p) & x(t-p+1) & \ldots & x(t-p+j-1)
\end{array}\right) \\
X_{t}=\left(\begin{array}{llll}
x(t) & x(t+1) & \ldots & x(t+j-1)
\end{array}\right)
\end{gathered}
$$

and $X_{t-p}^{c}$ and $X_{t}^{c}$ are respectively the past and future state matrices of the controller.

$$
\Gamma_{f}=\left(\begin{array}{c}
C \\
C A \\
\cdots \\
C A^{f-1}
\end{array}\right) \quad \text { and } \quad \Delta_{p}^{d}=\left(\begin{array}{llll}
A^{p-1} B & \cdots & A B & B
\end{array}\right)
$$

are respectively the extended observability matrix and the reversed extended controllability matrix of the deterministic part of the plant. $H_{f}^{d}$ is defined as

$$
H_{f}^{d}=\left(\begin{array}{cccc}
D & 0 & \cdots & 0 \\
C B & D & 0 & \cdots \\
\cdots & \cdots & \cdots & \cdots \\
C A^{f-2} B & \cdots & C B & D
\end{array}\right)
$$

and it corresponds to a lower triangular Toeplitz matrix containing the first $f$ Markov parameters of the deterministic part of the plant. Similar notations hold for the stochastic part i.e. $\Delta_{p}^{s}$ and $H_{f}^{s}$ and for the controller i.e. $\Gamma_{f}^{c}, \Delta_{p}^{c}$ and $H_{f}^{c}$.

Combinaisons of (2) and (3) yields

$$
\left\{\begin{array}{c}
x^{c l}(t+1)=A_{c l} x^{c l}(t)+B_{c l / r} r(t)+B_{c l / e} e(t) \\
y(t)=C_{c l} x^{c l}(t)+D_{c l / r} r(t)+D_{c l / e} e(t)
\end{array}\right.
$$

with

$$
x^{c l}(t)=\left(\begin{array}{c}
x(t) \\
x_{c}(t)
\end{array}\right)
$$

$A_{c l}, C_{c l}, B_{c l / r}, D_{c l / r}, B_{c l / e}$ and $D_{c l / e}$ are determined by the state space matrices $(A, B, C, D, K)$ and $\left(A_{c}, B_{c}, C_{c}, D_{c}\right)$. The order of this closed loop state space model is $n_{c l}=n+n_{c}$. This order should not be the minimal order $n_{c l}^{\text {min }}$ i.e. $n_{c l}^{\text {min }} \leq$ $n_{c l}$.

Similarly, a combinaison of (4) and (5) leads to an extended state space model of the closed loop:

$$
\left\{\begin{array}{c}
X_{t}^{c l}=A_{c l}^{p} X_{t-p}^{c l}+\Delta_{p}^{c l / r} R_{p}+\Delta_{p}^{c l / e} E_{p} \\
Y_{f}=\Gamma_{f}^{c l} X_{t}^{c l}+H_{f}^{c l / r} R_{f}+H_{f}^{c l / e} E_{f}
\end{array}\right.
$$

with

$$
\begin{array}{cc}
X_{t}^{c l}=\left(\begin{array}{c}
X_{t} \\
X_{t}^{c}
\end{array}\right) & \Gamma_{f}^{c l}=\left(\begin{array}{cc}
T_{f} \Gamma_{f} & T_{f} H_{f}^{d} \Gamma_{f}^{c}
\end{array}\right) \\
\Delta_{p}^{c l / r}=\left(\begin{array}{c}
\Delta_{p}^{d}\left(I_{p n_{u}}-H_{p}^{c} T_{p} H_{p}^{d}\right) \\
-\Delta_{p}^{c} T_{p} H_{p}^{d}
\end{array}\right) & H_{f}^{c l / r}=T_{f} H_{f}^{d} \\
\Delta_{p}^{c l / e}=\left(\begin{array}{c}
-\Delta_{p}^{d} H_{p}^{c} T_{p} H_{p}^{s}+\Delta_{p}^{s} \\
-\Delta_{p}^{c} T_{p} H_{p}^{s}
\end{array}\right) & H_{f}^{c l / e}=T_{f} H_{f}^{s}
\end{array}
$$


where $T_{f}=\left(I_{f n_{y}}+H_{f}^{d} H_{f}^{c}\right)^{-1}$. Notice that the well posed assumption of the closed loop ensures $\left(I_{f n_{y}}+H_{f}^{d} H_{f}^{c}\right)$ to be invertible. $A_{c l}^{p}$ can easily be expressed with matrices $A^{p}, A_{c}^{p}$, $H, \Delta$ and $\Gamma$.

To end this section let define the following projection operators as in [35]:

- The orthogonal projection $A /{ }^{B}$ of the row space of the matrix $A$ on the row space of the matrix $B$ :

$$
A /{ }^{B}=\mathscr{E}_{j}\left\{A B^{T}\right\}\left(\mathscr{E}_{j}\left\{B B^{T}\right\}\right)^{-1}
$$

The projection of $A$ onto the orthogonal complement of the row space of $B$ is defined as $A \Pi_{B}^{\perp}$

$$
A \Pi_{B}^{\perp}=A-A /{ }^{B} B
$$

- The oblique projection $A /{ }_{C}^{B}$ of the row space of the matrix $A$ on the row space of the matrix $B$ along the row space of $C$ :

$$
\begin{gathered}
A /{ }_{C}^{B}=\mathscr{E}_{j}\left\{A\left(\begin{array}{cc}
B^{T} & C^{T}
\end{array}\right)\right\}\left(\mathscr{E}_{j}\left\{\begin{array}{ll}
B B^{T} & B C^{T} \\
C B^{T} & C C^{T}
\end{array}\right\}\right)^{-1}\left(\begin{array}{c}
I \\
0
\end{array}\right) \\
A /{ }_{C}^{B}=\mathscr{E}_{j}\left\{A \Pi_{C}^{\perp} B^{T}\right\}\left(\mathscr{E}_{j}\left\{B \Pi_{C}^{\perp} B^{T}\right\}\right)^{-1}
\end{gathered}
$$

\section{PROPOSITION OF CLOSED LOOP IDENTIFICATION ALGORITHM}

Most of classical open loop subspace identification methods are based on the estimation of the extended observability matrix $\Gamma_{f}$. Whereas in a closed loop context the direct application of these methods gives biased estimates, the proposed indirect method allows an unbiased estimation of $\Gamma_{f}$ : this is the aim of the first subsection. A possible application algorithm for the estimation of a state matrices realization $(A, B, C, D)$ is given in the second subsection.

\section{A. Main results}

The first steps of the indirect algorithms by ([36]-[37]) and ([27], [29], [28]) are to estimate respectively $T_{f} \Gamma_{f}$ and $\Gamma_{f}$. Here the estimation of $\Gamma_{f}$ (or $T_{f} \Gamma_{f}$ ) will be done in a next step. We first concentrate on the estimation of a matrix describing the closed loop behavior i.e. the extended observability matrix of the closed loop $\Gamma_{f}^{c l}$. To this end the following result uses the standard procedure of subspace approach (see [35]) on (6).

Result 1:

Let define the following oblique projection matrix $\mathscr{O}_{f}^{c l}$ :

$$
\mathscr{O}_{f}^{c l}=Y_{f} /_{R_{f}}^{\Psi} \Psi \Pi_{R_{f}}^{\perp}
$$

where $\Psi$ is an instrumental variable matrix such that:

- $E_{f} / \Psi=0$

- the matrix $\mathscr{E}_{j}\left\{\left(\begin{array}{c}X_{t}^{c l} \\ R_{f}\end{array}\right)\left(\begin{array}{ll}\Psi^{T} & R_{f}^{T}\end{array}\right)\right\}$ has rank $\left(n_{c l}^{\text {min }}+\right.$ $\left.f n_{u}\right)$

Under assumptions stated in section II-A we have:

1) $\mathscr{O}_{f}^{c l}$ can be expressed with the extended observability matrix of the closed loop $\Gamma_{f}^{c l}$ and the state matrix $X_{t}^{c l}$

$$
\mathscr{O}_{f}^{c l}=\Gamma_{f}^{c l} X_{t}^{c l} /_{R_{f}}^{\Psi} \Psi \Pi_{R_{f}}^{\perp}
$$

2) The singular value decomposition (SVD) of $\mathscr{O}_{f}^{c l}$ is given by

$$
\begin{gathered}
\mathscr{O}_{f}^{c l}=\left(\begin{array}{cc}
\mathbb{U}_{1} & \mathbb{U}_{2}
\end{array}\right)\left(\begin{array}{cc}
\mathbb{S}_{1} & 0 \\
0 & 0
\end{array}\right)\left(\begin{array}{c}
\mathbb{V}_{1}^{T} \\
\mathbb{V}_{2}^{T}
\end{array}\right) \\
\mathscr{O}_{f}^{c l}=\mathbb{U}_{1} \mathbb{S}_{1} \mathbb{V}_{1}^{T}
\end{gathered}
$$

where $\mathbb{S}_{1} \in \mathbb{R}^{n_{c l}^{\min } \times n_{c l}^{\min }}$

3) $\Gamma_{f}^{c l}$ can be taken equal to $\Gamma_{f}^{c l}=\mathbb{U}_{1} T$ where $T$ is a similarity transformation.

Remark 1: The algorithm by [36]-[37] uses a similar oblique projection matrix: $Y_{f} /{ }_{U_{f}}^{\Psi} \Psi \Pi_{U_{f}}^{\perp}$. However this choice does not allow the estimation of the closed loop behavior.

The first condition on the instrumental variable matrix $\Psi$ must be guaranteed in order to obtain consistent estimation under the noise-corrupted context. Let remark that $A$ should note be stable which yields $E_{f} / U_{p} \neq 0$ as discussed in [2], thus $U_{p}$ don't have to be directly introduced in $\Psi$. Two possible choices are $\Psi=R_{p}$ or $\Psi=\left(\begin{array}{c}R_{p} \\ Y_{p}\end{array}\right)$.

The second condition on $\Psi$ (the rank condition) involves some conditions on the choice of the indexes $f$ and $p(f, p>$ $\left.n_{c l}^{\min }\right)$, the degree of persistence excitation and the closed loop complexity. According to [1], if $\Psi=R_{p}$ or $\Psi=\left(\begin{array}{c}R_{p} \\ Y_{p}\end{array}\right)$ this rank condition is generically satisfied provided that the exogenous input is persistently exciting enough.

Remark 2: Some weighting matrices are often added in (7) in order to include some well known subspace methods such that N4SID, PO-MOESP, CVA, IVM, etc. The MOESP type algorithms make use of the two previous instrumental variable matrices in (7).

Once the extended observability matrix known, we have to find $\Gamma_{f}$. From (6) it is easy to rewrite $\mathscr{O}_{f}^{c l}$ as

$$
\mathscr{O}_{f}^{c l}=T_{f} \Gamma_{f} X_{t} /_{R_{f}}^{\Psi} \Psi \Pi_{R_{f}}^{\perp}+T_{f} H_{f}^{d} \Gamma_{f}^{c} X_{t}^{c} / \Psi R_{f} \Psi \Pi_{R_{f}}^{\perp}
$$

which gives

$$
\Gamma_{f} X_{t} /_{R_{f}}^{\Psi} \Psi \Pi_{R_{f}}^{\perp}=T_{f}^{-1}\left(\mathscr{O}_{f}^{c l}-T_{f} H_{f}^{d} \Gamma_{f}^{c} X_{t}^{c} /_{R_{f}}^{\Psi} \Psi \Pi_{R_{f}}^{\perp}\right)
$$

with

$$
\Gamma_{f}^{c} X_{t}^{c}=U_{f}+H_{f}^{c} Y_{f}-R_{f}
$$

To compute $\Gamma_{f}$ we need to determine matrices $T_{f} H_{f}^{d}$ and $T_{f}$. From (6) we get

$$
Y_{f} /{ }^{Z} Z=\Gamma_{f}^{c l} X_{t}^{c l} /{ }^{Z} Z+T_{f} H_{f}^{d} R_{f}
$$

where $Z=\left(\begin{array}{c}R_{f} \\ \Psi\end{array}\right)$. With $\left(\widehat{\Gamma_{f}^{c l}}\right)^{\perp}$ computed from the previous result we find:

$$
\left(\widehat{\Gamma_{f}^{l} l}\right)^{\perp} Y_{f} /{ }^{Z} Z=\left(\widehat{\Gamma_{f}^{c l}}\right)^{\perp} T_{f} H_{f}^{d} R_{f}
$$

with

$$
T_{f} H_{f}^{d}=\left(\begin{array}{cccc}
L_{0} & 0 & \cdots & 0 \\
L_{1} & L_{0} & \ddots & \vdots \\
\vdots & \ddots & \ddots & 0 \\
L_{f-1} & \cdots & L_{1} & L_{0}
\end{array}\right)
$$


where $L_{0}=D_{c l / r}$ and $L_{i}=C_{c l} A_{c l}^{i-1} B_{c l / r}$. These $f$ elements $L_{i} \in \mathbb{R}^{n_{y} \times n_{u}}$ can easily be extracted using the procedure given in [36].

Once $\widehat{T_{f} H_{f}^{d}}$ is known, it is easy to get an estimated of $T_{f}$ :

$$
\widehat{T_{f}}=I_{f n_{y}}-\widehat{T_{f} H_{f}^{d}} H_{f}^{c}
$$

By using previous estimations, the following result allows the computation of the extended observability matrix $\Gamma_{f}$ of the plant model.

\section{Result 2:}

Let define the following matrix $\mathscr{O}_{f}$ :

$$
\mathscr{O}_{f}=T_{f}^{-1}\left(\mathscr{O}_{f}^{c l}-\left.T_{f} H_{f}^{d} \Gamma_{f}^{c} X_{t}^{c}\right|_{R_{f}} ^{\Psi} \Psi \Pi_{R_{f}}^{\perp}\right)
$$

Under the assumption stated in part II-A and in the previous result we have:

1) The matrix $\mathscr{O}_{f}$ can be expressed with the extended observability matrix of the plant $\Gamma_{f}$ and the state matrix $X_{t}$

$$
\mathscr{O}_{f}=\Gamma_{f} X_{t} /{ }_{R_{f}}^{\Psi} \Psi \Pi_{R_{f}}^{\perp}
$$

2) The SVD of $\mathscr{O}_{f}$ is given by

$$
\begin{gathered}
\mathscr{O}_{f}=\left(\begin{array}{cc}
\mathbb{U}_{1}^{\prime} & \mathbb{U}_{2}^{\prime}
\end{array}\right)\left(\begin{array}{cc}
\mathbb{S}_{1}^{\prime} & 0 \\
0 & 0
\end{array}\right)\left(\begin{array}{c}
\mathbb{V}^{\prime T} \\
\mathbb{V}^{\prime}{ }_{2}^{T}
\end{array}\right) \\
\mathscr{O}_{f}=\mathbb{U}^{\prime}{ }_{1} \mathbb{S}_{1}^{\prime} \mathbb{V}^{\prime}{ }_{1}^{T}
\end{gathered}
$$

where $\mathbb{S}_{1}^{\prime} \in \mathbb{R}^{n \times n}$,

3) $\Gamma_{f}$ can be taken equal to $\Gamma_{f}=\mathbb{U}^{\prime}{ }_{1} T$ where $T$ is a similarity transformation.

Remark 3: It is possible to estimate a matrix $\widehat{X}_{t}=$ $X_{t} /{ }_{R_{f}}^{\Psi} \Psi \Pi_{R_{f}}^{\perp}=T^{-1} \mathbb{S}_{1}^{\prime} \mathbb{V}^{\prime}{ }_{1}^{T}$. This can be interpreted as the state sequence estimated by a bank of non steady state Kalman filters ([35]).

Remark 4: It should be noted that results 1 and 2 are based on classical subspace procedures ([35]) and algebraic computations applicable to MIMO processes, thus these results are also applicable to this type of processes.

Remark 5: In the previous result the Markov parameters of the controller are needed: in (9) and (10). It should be noted that (5) can be rewritten as $\left(U_{f}-R_{f}\right)=\Gamma_{f}^{c} X_{t}^{c}-H_{f}^{c} Y_{f}$. This equation is free noise, thus the matrix $H_{f}^{c}$ can easily be estimated without bias making use an open loop subspace algorithm.

\section{B. Closed loop identification algorithm}

In this section we propose the estimation of a realization of state space matrices $(A, B, C, D)$. This can be realized with the estimated state sequence $\widehat{X}_{t}$, however we present the solution making use of the shift invariance structure of the extended observability matrix $\Gamma_{f}$.

The basic steps of the proposed algorithm are as follows: step 1:Compute the LP decomposition

$$
\left(\begin{array}{c}
R_{f} \\
\Psi \\
Y_{f}
\end{array}\right)=\left(\begin{array}{ccc}
M_{11} & 0 & 0 \\
M_{21} & M_{22} & 0 \\
M_{31} & M_{32} & M_{33}
\end{array}\right)\left(\begin{array}{c}
N_{1}^{T} \\
N_{2}^{T} \\
N_{3}^{T}
\end{array}\right)
$$

which gives the first oblique projection

$$
\mathscr{O}_{f}^{c l}=M_{32} N_{2}^{T}
$$

step 2:Compute the SVD (8) and extract $\Gamma_{f}^{c l}$

$$
\Gamma_{f}^{c l}=\mathbb{U}_{1}
$$

step 3:Estimate $T_{f} H_{f}^{d}$ and $T_{f}$

step 4:Compute the LP decomposition

$$
\left(\begin{array}{c}
R_{f} \\
\Psi \\
T_{f} H_{f}^{d} \Gamma_{f}^{c} X_{t}^{c}
\end{array}\right)=\left(\begin{array}{ccc}
R_{11} & 0 & 0 \\
R_{21} & R_{22} & 0 \\
R_{31} & R_{32} & R_{33}
\end{array}\right)\left(\begin{array}{c}
Q_{1}^{T} \\
Q_{2}^{T} \\
Q_{3}^{T}
\end{array}\right)
$$

which gives the second oblique projection

$$
\mathscr{O}_{f}=T_{f}^{-1}\left(\mathbb{U}_{1} \mathbb{S}_{1} \mathbb{V}_{1}^{T}-R_{32} Q_{2}^{T}\right)
$$

step 5:Compute the SVD (11) and extract $\Gamma_{f}$

$$
\Gamma_{f}=\mathbb{U}_{1}^{\prime}
$$

step 6:The system matrices $A$ and $C$ can be estimated by

$$
\begin{gathered}
C=\Gamma_{f}\left(1: n_{y},:\right) \\
A=\left(\underline{\Gamma}_{f}^{T} \underline{\Gamma}_{f}\right)^{-1} \underline{\Gamma}_{f}^{T} \bar{\Gamma}_{f}
\end{gathered}
$$

with $\underline{\Gamma}_{f}=\Gamma_{f}\left(1:(f-1) n_{y},:\right)$ and $\bar{\Gamma}_{f}=\Gamma_{f}\left(n_{y}+1\right.$ : $\left.f n_{y},:\right)$.

$B$ and $D$ can be estimated from the following equation

$$
\left(T_{f}^{-1} \Gamma_{f}^{c l}\right)^{\perp} T_{f}^{-1} Y_{f} /^{Z} Z=\left(T_{f}^{-1} \Gamma_{f}^{c l}\right)^{\perp} H_{f}^{d}(B, D) R_{f}
$$

using the procedure given in [35].

Remark 6: Note that the absence of feedback corresponds to the case $H_{f}^{c}=0, \Gamma_{f}^{c}=0, R_{f}=U_{f}$ and $R_{p}=U_{p}$. As a consequence, in an open loop context the proposed algorithm can be reduced to steps 1,2 and 6 . This corresponds to the open loop MOESP type algorithms: with $\Psi=U_{p}$ our algorithm is equivalent to the PI-MOESP algorithm, with $\Psi=\left(\begin{array}{c}U_{p} \\ Y_{p}\end{array}\right)=$ our algorithm is equivalent to the POMOESP algorithm.

\section{NUMERICAL EXAMPLE}

In this section we consider a fifth order plant presented in [38] and used as a benchmark problem by [36] for closed loop subspace identification. This example is also adopted in [24], [20], [13], [12], [21] for comparison.

The transfert functions of the plant and controller are respectively given by

$$
G(q)=10^{-3} q^{-1} \frac{0.98+12.99 q^{-1}+18.59 q^{-2}+3.3 q^{-3}-0.02 q^{-4}}{1-4.4 q^{-1}+8.09 q^{-2}-7.83 q^{-3}+4 q^{-4}-0.86 q^{-5}}
$$




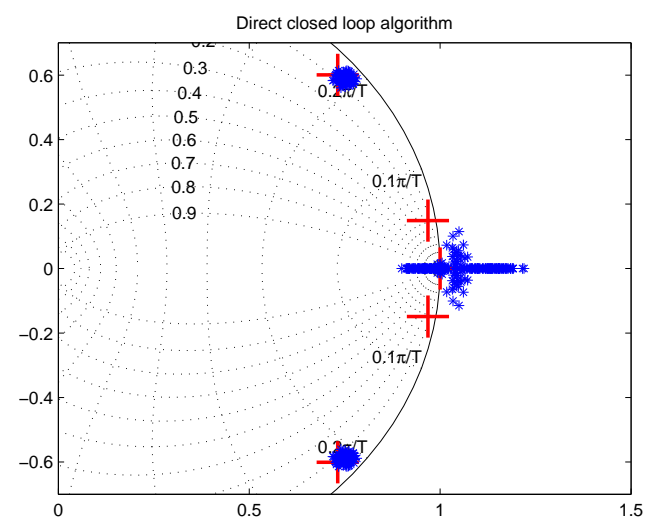

Fig. 2. The eigenvalues of estimated A matrix with direct approach: * estimated pole, + system pole.

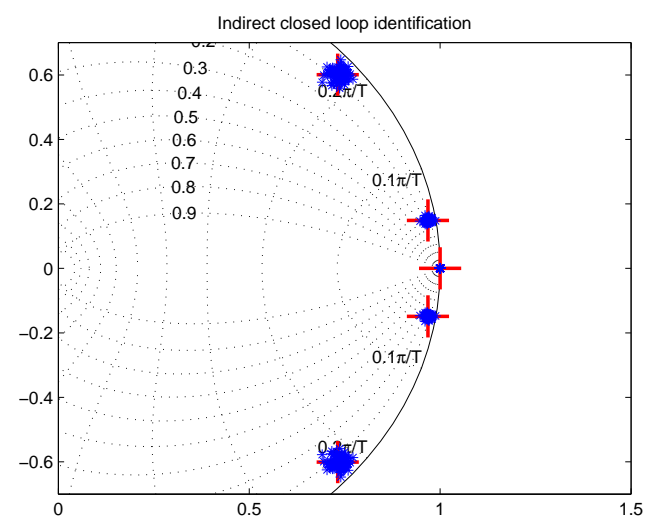

Fig. 3. The eigenvalues of estimated A matrix with our indirect approach: * estimated pole, + system pole.

$$
C(q)=\frac{0.63-2.083 q^{-1}+2.8222 q^{-2}-1.865 q^{-3}+0.4978 q^{-4}}{1-2.65 q^{-1}+3.11 q^{-2}-1.75 q^{-3}+0.39 q^{-4}}
$$

The exogenous input $r(t)$ is a gaussian white noise sequence with variance 1 . The innovation $e(t)$ is a gaussian white noise with variance $1 / 9$ and the noise model is given by

$$
H(q)=\frac{1-2.1 q^{-1}+1.45 q^{-2}-0.315 q^{-3}-0.0146 q^{-4}+0.0034 q^{-5}}{1-4.4 q^{-1}+8.09 q^{-2}-7.83 q^{-3}+4 q^{-4}-0.86 q^{-5}}
$$

The number of columns in Hankel matrices is $j=1200$ and we generate 100 data sets, each time with the same exogenous input but with different noise sequences. We choose $f=20$ and the following instrumental variable $\Psi$

$$
\Psi=\left(\begin{array}{c}
R_{p} \\
Y_{p}
\end{array}\right)
$$

where $R_{p}$ and $Y_{p}$ are built with $p=f=20$. The Markov parameters of the controller are supposed to be known.

Some estimates of the poles are shown in Figs. 2 and 3: Fig. 2 corresponds to the N4SID algorithm proposed by MATLAB, Fig. 3 corresponds to our indirect algorithm. We can see that our indirect method looks better than the other in spite of one remark: poles $0.7319 \pm 0.6007 i$ are difficult to estimate, the estimation variance is higher (Fig 3).

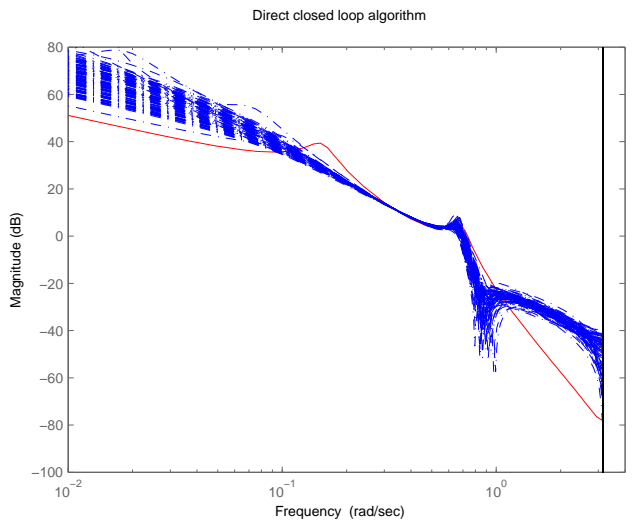

Fig. 4. The Bode magnitude plot of direct approach.

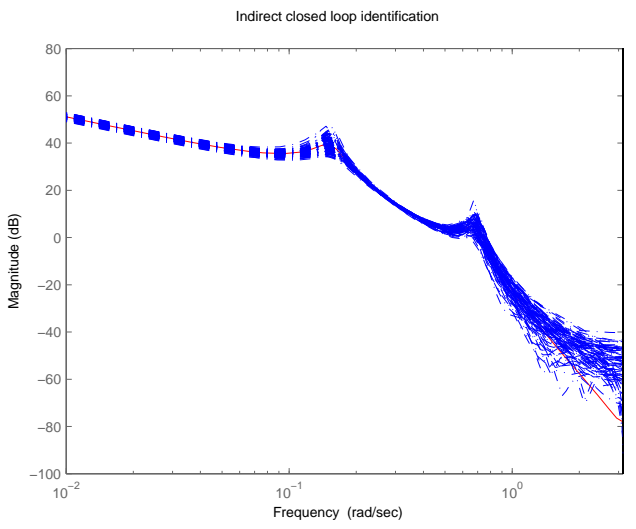

Fig. 5. The Bode magnitude plot of our indirect approach.

Estimates of the frequency response are shown in Fig. 4 to 7: Fig. 4 corresponds to the N4SID algorithm proposed by MATLAB, Fig. 5 corresponds to our indirect method, Fig. 6 corresponds to the method proposed in [36]-[37] (with $p=f=10-$ as proposed in these papers) and Fig. 7 corresponds to the method proposed in [29] (with $p=f=20$ - the best choice after several tests). Here again, our indirect method works well: all the methods match with the frequency response of the real system at low and medium frequency.

\section{CONCLUSION}

In this paper, an indirect closed-loop identification algorithm is developed using the subspace identification culture. The key idea was to estimate a characteristic matrix of the closed loop behavior and then to extract a characteristic matrix of the plant. Notice that two LQ decompositions and two SVD are needed and the algorithm allows the estimation of the closed loop complexity and plant complexity. In this paper only the deterministic part of the plant is identified, follow-up paper will concentrate on the stochastic part.

\section{REFERENCES}

[1] D. Bauer and M. Jansson. "Analysis of the asymptotic properties of the MOESP type of subspace algorithms". Automatica, 36 (4),497-509, 2000 . 


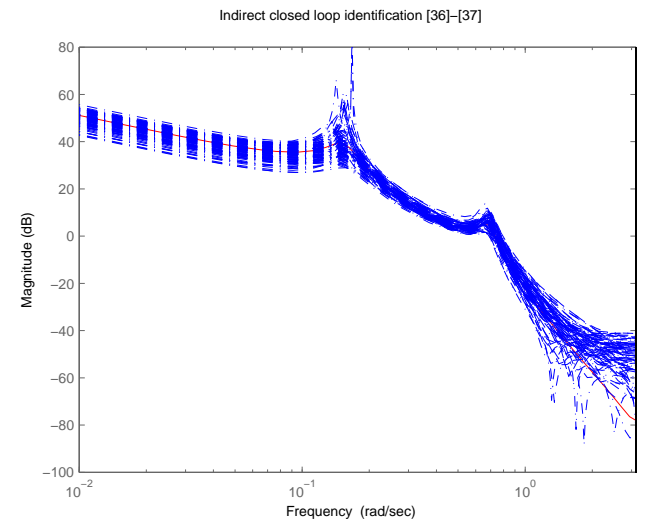

Fig. 6. The Bode magnitude plot of indirect approach of [36]-[37].

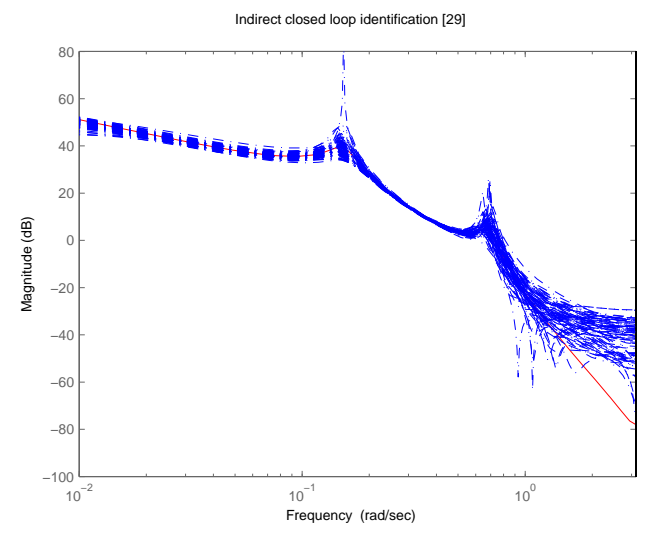

Fig. 7. The Bode magnitude plot of indirect approach of [29].

[2] A. Chiuso and G. Picci. "Consistency analysis of some closed loop subspace identification methods". Automatica, 41 (3),377-391, 2005.

[3] A. Chiuso and G. Picci. "Prediction error vs subspace methods in closed loop identification". IFAC World Congress, Prague, 2005.

[4] A. Chiuso. "Asymptotic variance of closed-loop subspace identification methods". Transactions Automatic Control, 51 (8), 1299-1314, 2006.

[5] A. Chiuso. "Asymptotic equivalence of certain closed loop subspace identification methods". Proceedings $14^{\text {th }}$ IFAC Symposium on System Identification, Newcastle, 2006.

[6] A. Chiuso. "The role of vector auto regressive modeling in predictor based subspace identification". Automatica, 43 (6),10341048, 2007.

[7] C.T. Chou and M. Verhaegen. "Closed loop Identification using Canonical Correlation Analysis". European Control Conference, Karlsruhe, 1999.

[8] B. Codrons and B.D.O. Anderson and M. Gevers. "Closed loop identification with an unstable or nonminimum phase controller". Automatica, 38 (12),2127-2137, 2002.

[9] W. Favoreel and B. De Moor and P. Van Overschee. "Subspace state space system identification for industrial processes". Journal of Process Control, 10,149-155, 2000.

[10] U. Forssell and L. Ljung. "Closed loop identification revisited". Automatica, 35, 1215-1241, 1999.

[11] M. Gilson and P.M.J. Van Den Hof. "Instrumental variable methods for closed loop system identification". Automatica, 41 (2),241-249, 2005.

[12] M. Gilson and G. Mercere. "Subspace based optimal IV method for closed loop system identification". Proceedings $14^{\text {th }}$ IFAC Symposium on System Identification, Newcastle, 2006.

[13] B. Huang and and S.X. Ding and S.J. Qin. "Closed-loop subspace identification: an orthogonal projection approach". Journal of Process Control, 15 ,53-66, 2005.
[14] B. Huang and R. Kadali. "Dynamic Modeling, Predictive Control and Performance Monitoring. A Data-driven Subspace Approach". Springer, Berlin, 2008.

[15] M. Jansson. "Subspace identification and ARX modeling". 13 ${ }^{\text {th }}$ IFAC Symposium on System Identification, Rotterdam, 2003.

[16] M. Jansson. "A New Subspace Identification Method for Open and Closed Loop Data". 16 ${ }^{\text {th }}$ IFAC World Congress, Prague, 2005.

[17] T. Katayama and T. Yamamoto. "A State-Space Model identification of closed loop System Based on Subspace Method". American Control Conference, Seattle, 1995.

[18] T. Katayama. "Subspace methods for system identification". SpringerVerlag, 2005.

[19] T. Katayama and H. Kawauchi and G. Picci. "Subspace identification of closed loop systems by the orthogonal decomposition method". Automatica, 43 (9), 863-872, 2005.

[20] T. Katayama and H. Tanaka and T. Enomoto. "A simple subspace identification method of closed-loop systems using orthogonal decomposition". IFAC World Congress, Prague, 2005.

[21] T. Katayama and H. Tanaka. "An approach to closed loop subspace identification by orthogonal decomposition". Automatica, 43 (9), 16231630, 2007.

[22] T. Katayama and H. Ase "A New Subspace Identification Method for closed loop Systems using Measurable Disturbance". IFAC World Congress, Seoul, 2008.

[23] W. Lin and S.J. Qin and L. Ljung. "On consistency of closed loop subspace identification with innovation estimation". Proceedings $43^{\text {rd }}$ Control and Decision Conference, Bahamas, 2004.

[24] W. Lin and S. Qin and L. Ljung "Comparisons of subspace identification methods for systems operating in closed loop". IFAC World Congress, Prague, 2005.

[25] L. Ljung anf T. Mc Kelvey. "Subspace identification from closed loop data". Signal Processing, 52, 209-215, 1996.

[26] L. Ljung. "System identification: theory for the user". Prentice Hall, 1999.

[27] H. Oku and T. Fujii. "Direct subspace model identification of LTI systems operating in closed loop". Proceedings $43^{\text {rd }}$ Control and Decision Conference, Bahamas, 2004

[28] H. Oku and Y. Ogura and T. Fujii. "Closed loop subspace model identification based on QR-factorization". Proceedings $17^{\text {th }}$ International Symposium on Mathematical Theory of Networks and Systems, Kyoto, 2006.

[29] H. Oku and H. Tanaka. "Closed loop Subspace Model Identification". Institute of Systems, Control and Information Engineers, 50 (3),106111, 2006.

[30] S.J. Qin and L. Ljung. "Closed loop subspace identification with innovation estimation". $13^{\text {th }}$ IFAC Symposium on System Identification, Rotterdam, 887-892, 2003.

[31] S.J. Qin and W. Lin and L. Ljung. "A novel subspace identification approach with enforced causal models". Automatica, 41, 2043-2053, 2005.

[32] S.J. Qin. "An overview of subspace identification". Computers and chemical engineering, 30, 1502-1513, 2006.

[33] T. Soderstrom and P. Stoica. "System identification". Prentice Hall, 1989.

[34] P.M.J. Van Den Hof and R.J.P. Schrama. "An indirect method for transfer function estimation from closed loop data". Automatica, 29 (6), 1523-1527, 1993.

[35] P. Van Overschee and B. De Moor. "Subspace identification for linear systems. Theory, implementation applications". Kluver Academic Publishers, Leuven, 1996.

[36] P. Van Overschee and B. De Moor. "Closed loop subspace system identification". Technical report - Katholieke Universiteit Leuven, 1996.

[37] P. Van Overschee and B. De Moor. "Closed loop subspace system identification". Proceedings $36^{\text {th }}$ Control and Decision Conference, San Diego, 1848-1853, 1997.

[38] M. Verhaegen. "Application of a subspace model identification technique to identify LTI systems operating in closed loop". Automatica, 29 (4), 1027-1040, 1993.

[39] J. Wang and S.J. Qin. "Closed loop subspace identification using the parity space". Automatica, 42, 315-320, 2006. 